\title{
Spatial distribution of non-linear interdependency measures for focal hemisphere identification in epileptic patients from interictal intracranial EEG
}

\author{
Mario Pannunzi*1, Stefano Ferraina ${ }^{2}$ and Paolo Del Giudice ${ }^{1}$
}

\author{
Address: ${ }^{1}$ Department of Technologies and Health, Italian National Institute of Health, Rome, Italy; INFN, sezione Rome, Italy and ${ }^{2}$ Department \\ of neurophysiology, Rome, Italy \\ Email: Mario Pannunzi* - mario.pannunzi@gmail.com \\ * Corresponding author
}

from Eighteenth Annual Computational Neuroscience Meeting: CNS*2009

Berlin, Germany. 18-23 July 2009

Published: 13 July 2009

BMC Neuroscience 2009, 10(SuppI I):P8I doi:10.1 I86/I47|-2202-I0-SI-P8|

This abstract is available from: http://www.biomedcentral.com//47I-2202//0/SI/P8I

(C) 2009 Pannunzi et al; licensee BioMed Central Ltd.

We study performance, stability and spatial distribution of three previously proposed $[1,2]$ non-linear measures of interdependency between time series (named $H, M$ and $S$ ) used to classify, from interictal recordings, the epileptogenic hemisphere in patients with drug-resistant mesial temporal lobe epilepsy (MTLE). Two electrodes penetrate the hippocampal region in the two hemispheres and include 10 recording contacts each. We consider only data recorded during interictal periods [3]. All measures are introduced through the reconstruction of a "state-space" for the recorded signals, using the widely adopted "embedding" approach, and quantify in different ways the average distance between neighbours in phase space for one signal and the distance between the corresponding equal-time partners in the other signal. One hemisphere is classified as focal if the average between-contacts interdependency value is significantly greater than the one for the other hemisphere. We investigate the dependence of the interdependency measures and associated performances on the inter-electrode distance, on the relevant parameters and non-stationarities across interictal periods. Two of the three measures ( $H$ and $M$ ) provide good and similar classification performances, as well as similar spatial distributions. For $M$, ten cases are correctly classified, one case is incorrectly classified and for four cases, $M$ values are statistically indistinguishable for the two hemispheres. For the correctly classified cases, $M$ shows long-range between-contacts interdependencies for the focal hemisphere (see Figure 1). We also show how interdependencies vary inside one interictal period and between different interictal periods. The role of the parameters entering the analysis is systematically studied to provide heuristic criteria for their choice. Two of the studied nonlinear measures are found to be adequate for the classification of the focal hemisphere. The observed long-range interdependency for focal hemispheres is consistent with the expected propensity of pathological nervous tissue to be entrained in paroxysmal synchronous activity. We suggest that the observed non-stationarity of interdependencies across interictal periods could be used to improve the classification performance.

\section{Acknowledgements}

We are grateful to R.G. Andrzejak for fruitful discussion and for helpful discussions at various stages of the work.

\section{References}

I. Arnhold J, Grassberger P, Lenhertz K, Elger CE: A robust method for detecting interdependences: application to intracially recorded EEG. Physica D 1999, 134:419-430.

2. Andrzejak RG, Kraskov A, Stogbauer H, Mormann F, Kreuz T: Bivariate surrogate techniques: Necessity, strengths and caveats. Phys Rev E 2003, 68:066202.

3. Recordings from 15 epileptic patients subject to invasive presurgical diagnostics performed at the Department of Epileptology of the University of Bonn, Germany. 

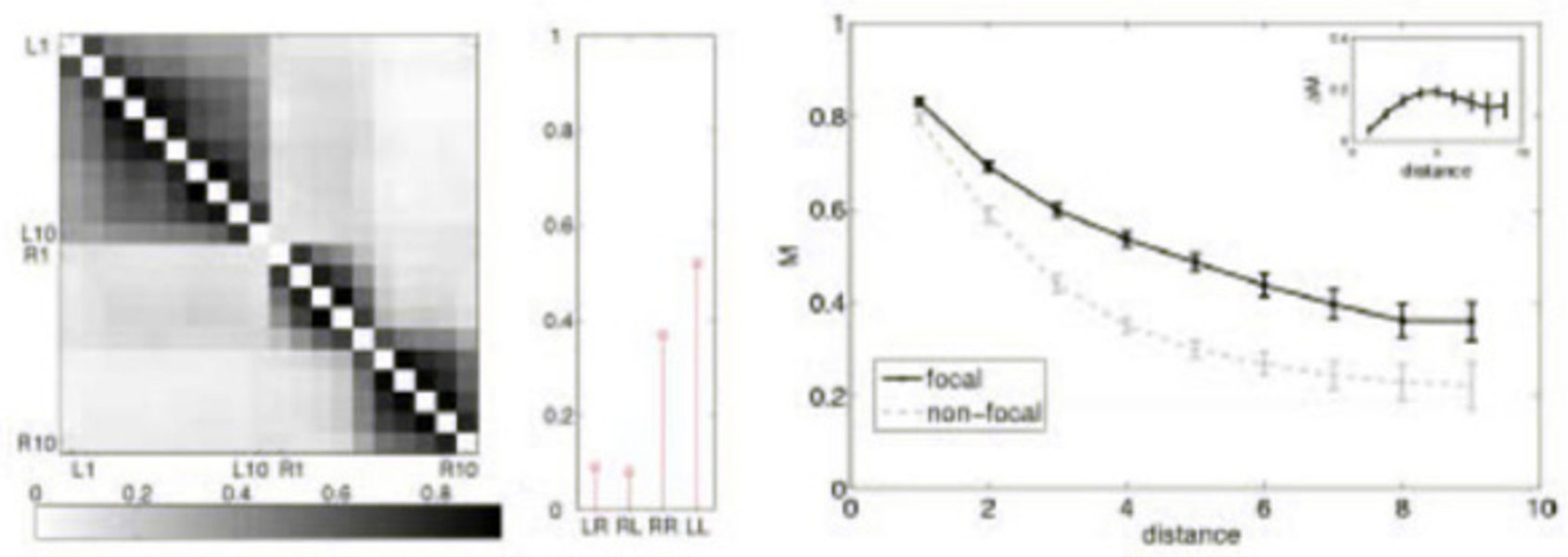

\section{Figure I}

Left: Spatial distribution of the measure $M$, for a case of correct localization of the focal hemisphere. Stem-plots: Averages of the measure in the four quadrants LL, LR, RL and RR. Right: $M$ vs inter-contact distance, averaged over all the contact pairs and over all the correct classification cases (I0) at a given distance. Horizontal dashed line: average $M$ values for cross-hemispheres interdependencies.

Publish with Bio Med Central and every scientist can read your work free of charge

"BioMed Central will be the most significant development for disseminating the results of biomedical research in our lifetime."

Sir Paul Nurse, Cancer Research UK

Your research papers will be:

- available free of charge to the entire biomedical community

- peer reviewed and published immediately upon acceptance

- cited in PubMed and archived on PubMed Central

- yours - you keep the copyright 